\title{
Aplicación de un nuevo modelo metodológico para la introducción al análisis estadístico en el Grado en Geografía y Gestión del Territorio
}

\author{
Application of a new \\ methodological model for the \\ introduction to statistical analysis \\ in the Degree in Geography \\ and Territorial Management
}

ESPERANZA SÁNCHEZ RODRÍGUEZ

ORCID: https://orcid.org/0000-0001-7446-4236

Universidad de Sevilla

Departamento de Geografía Física

y Análisis Geográfico Regional

esanchez@us.es

DOI: http:T/dx.doi.org/10.12795/9788447221912.004 Pp.: 108-131 
Se presenta el proceso llevado a cabo, en el marco del Curso General de Docencia Universitaria del Programa FIDOP de la Universidad de Sevilla, para la mejora docente en la asignatura Estadística y Bases de Datos del Grado en Geografia y Gestión del Territorio, trabajando contenidos correspondientes a la introducción al análisis estadístico. Para despertar el interés de los alumnos se parte de una cuestión de actualidad como es, en la época electoral que vivíamos, el barómetro del CIS y su estimación de voto. Se ha construido el mapa de contenidos y se ha diseñado una secuencia de actividades que implica una metodología participativa que requiere realizar diversas actividades intelectuales en clase, muy diferente del modelo transmisivo utilizado hasta entonces. Los resultados del aprendizaje y la metodología se han evaluado y se consideran positivos. Sin embargo, estimamos que la principal aportación de este proceso ha sido el incremento de la motivación de alumnos y profesora.

Palabras clave: Estadística y bases de datos, Geografia y Gestión del Territorio, Docencia universitaria, Experimentación docente universitaria.

\section{Abstract}

Within the framework of the General Course of University Teaching, a process for the teaching improvement in the subject Statistics and Data Bases (Degree in Geography and Territorial Management) has been carried out; it has addressed contents corresponding to the introduction to the statistical analysis. To awaken the interest of the students, we started from a current issue as the barometer of the CIS and its vote estimation. The content map was constructed and a sequence of activities was designed; it implies a participatory methodology that requires students to perform different intellectual tasks in class and is very different from the transmissive model used until then. The learning outcomes and methodology have been evaluated, with very satisfactory results. However, we believe that the main contribution of this process has been to increase both students and teacher motivation.

Keywords: Statistics and databases, Geography and Territorial Management, University teaching, University teaching experimentation

Jornadas de Formación e Innovación Docente del Profesorado | № 2 (2019) Esta obra se distribuye con la licencia Creative Commons Reconocimiento-NoComercial-SinObraDerivada Internacional (CC BY-NC-ND 4.0.) 


\section{Contexto}

La experiencia que aquí se describe se ha desarrollado en el grupo 2 de la asignatura Estadística y Bases de Datos, obligatoria de 6 créditos (1.5 teóricos y 4.5 prácticos) de 2o curso, que se imparte conjuntamente en el Grado en Geografia y Gestión del Territorio y el Doble Grado en Geografía y Gestión del Territorio e Historia durante el segundo cuatrimestre del curso 2018-19.

El objetivo de la asignatura es iniciar a los estudiantes en las técnicas y herramientas necesarias para la organización, modelado, consulta y explotación estadística de la información relativa a las variables geográficas. La asignatura tiene dos partes bien diferenciadas: bases de datos y estadística. El primer ciclo de mejora dentro del Curso General de Docencia Universitaria (CGDU) se desarrolló sobre contenidos de bases de datos; el momento de llevar a la práctica el segundo ciclo de mejora se correspondió con el inicio del bloque de estadística, por lo que se trabajaron los contenidos de "Introducción al análisis estadístico", es decir, los conceptos básicos y el análisis inicial de datos (distribuciones de frecuencia y representaciones gráficas).

El grupo está formado por 25 alumnos, aunque sólo asisten a clase con regularidad unos 16, de los que 8 son repetidores. En el momento de su desarrollo todos los alumnos tenían ya experiencia en ciclos de mejora, puesto que los no repetidores habían participado en las sesiones correspondientes de otra asignatura (Climatología y Biogeografia) que se llevaron a cabo un poco antes, y los repetidores lo hicieron el curso anterior en otra asignatura obligatoria (Geomorfología e Hidrología). Debemos señalar que, al iniciar el ciclo de mejora, el conjunto de alumnos se caracterizaba por su división en grupos aislados que apenas se comunicaban y que se sentaban muy alejados entre sí. El grupo de no repetidores era muy cerrado, no conocía ni se interesaba por los demás; entre los 
repetidores, un grupo de cuatro estudiantes si se conocía, se sentaban juntos y hacían comentarios entre ellos, pero los demás iban por libre; el grupo se completa con una estudiante Erasmus francesa que, aunque habla español perfectamente, parecía tímida y se sentaba siempre sola. Aunque inicialmente se pensó este ciclo para realizarlo en cuatro sesiones (8 horas de clase), esta experiencia se desarrolló en cinco sesiones de dos horas, en el horario habitual de la asignatura: miércoles de 18 a 20 y jueves de 17 a 19.

\section{Diseño previo del ciclo de mejora en el aula}

Tal como está establecido en el contexto del CGDU, el diseño del ciclo de mejora incluye la construcción del mapa de contenidos y problemas, la definición del modelo metodológico posible, el diseño de la secuencia de actividades a realizar y el planteamiento del cuestionario inicial/final que se utiliza para la evaluación tanto de las ideas previas como del aprendizaje de los estudiantes.

\section{Mapa de contenidos}

El mapa de contenidos se presenta en la Figura 1. El problema en torno al cual se construye es el Barómetro del CIS (Centro de Investigaciones Sociológicas) como fuente estadística para el estudio de la sociedad española. Empezamos preguntándonos qué es el barómetro y para qué sirve, lo que permite trabajar el contenido actitudinal de valorar el conocimiento científico y sus fuentes. Seguiremos preguntándonos a quien se le hacen las preguntas de la encuesta del barómetro, para llegar a los conceptos básicos del método estadístico: individuo, muestra, población y las diferencias entre la estadística descriptiva y la inferencial. 
A continuación pasaremos a examinar las preguntas de la encuesta del CIS, lo que lleva al concepto de variable estadística. Veremos que hay distintos tipos de preguntas que se corresponden con diferentes tipos de variables, que dan niveles de información variados (variables cuantitativas/cualitativas, escalas nominal, ordinal, de intervalos y de proporciones). Es importante saber diferenciar el tipo de variable con el que se trabaja porque de ello dependen las técnicas estadísticas que se pueden o deben aplicar, por lo que en este punto se incluye también el contenido procedimental: “¿cómo diferenciar los tipos de variables?"

Para el análisis de una variable estadística, el primer paso es resumir la información que proporcionan. Eso se hace de dos formas diferentes: a través de gráficos y a través de tablas de frecuencias (absolutas, relativas y acumuladas, y agrupadas o sin agrupar). Hay que saber qué son, pero también cómo se hacen y cómo se interpretan (contenido procedimental) en función del tipo de variable.

Para finalizar incluimos el aspecto ético del tratamiento estadístico de los datos (contenido actitudinal), que se relaciona con la forma de seleccionar la muestra, la forma de resumir los datos (gráficos o tablas de frecuencia) y la forma de presentar los resultados.

Jornadas de Formación e Innovación Docente del Profesorado | № 2 (2019) Esta obra se distribuye con la licencia Creative Commons 


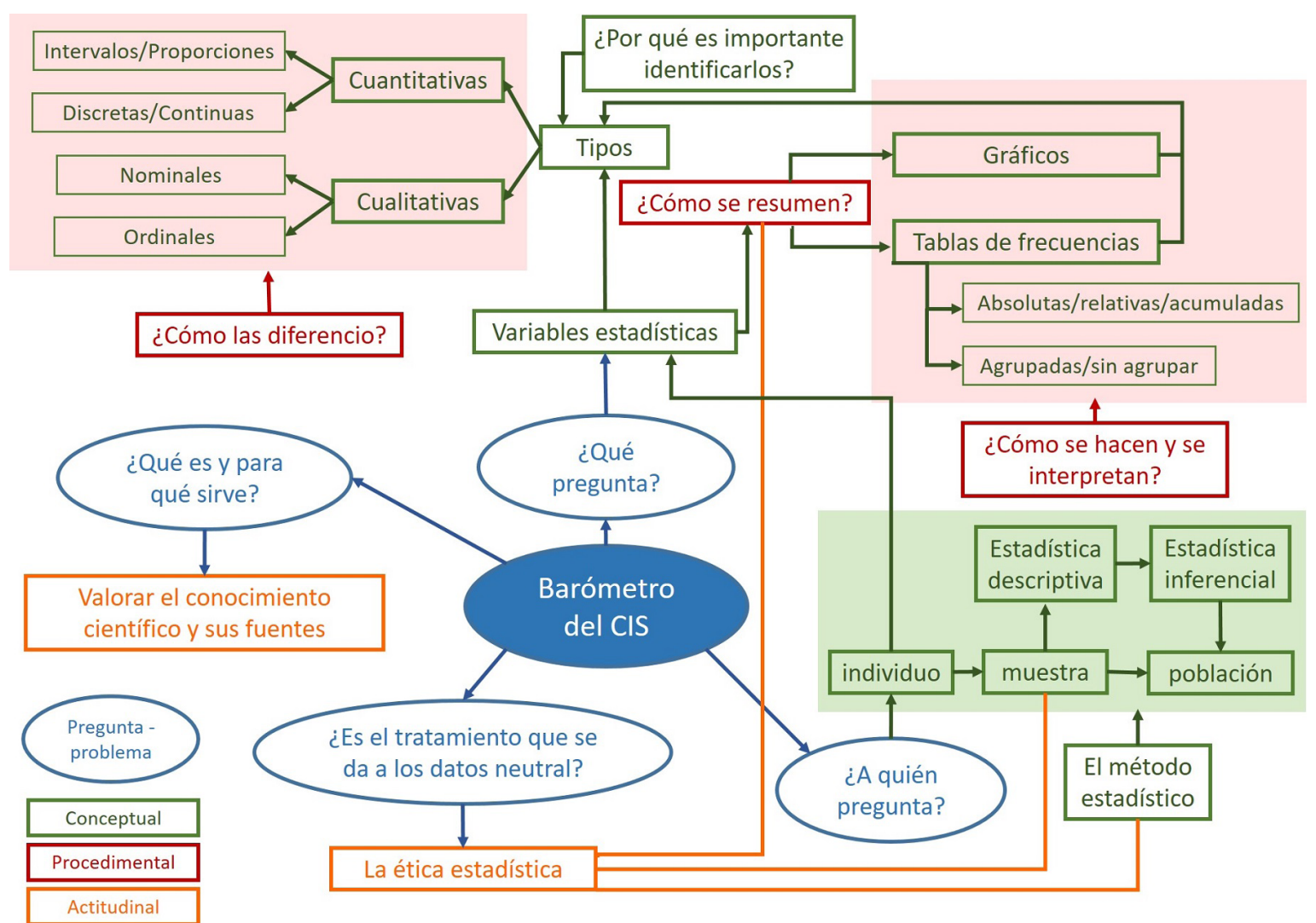

Figura 1. Mapa de contenidos

Jornadas de Formación e Innovación Docente del Profesorado I № 2 (2019)

cc (i) $\odot$ Esta obra se distribuye con la licencia Creative Commons 


\section{Modelo metodológico posible y secuencia de actividades}

Tras la experiencia obtenida en la aplicación del primer ciclo de mejora, el modelo metodológico que considero posible aplicar en este segundo ciclo se presenta en la Figura 2.

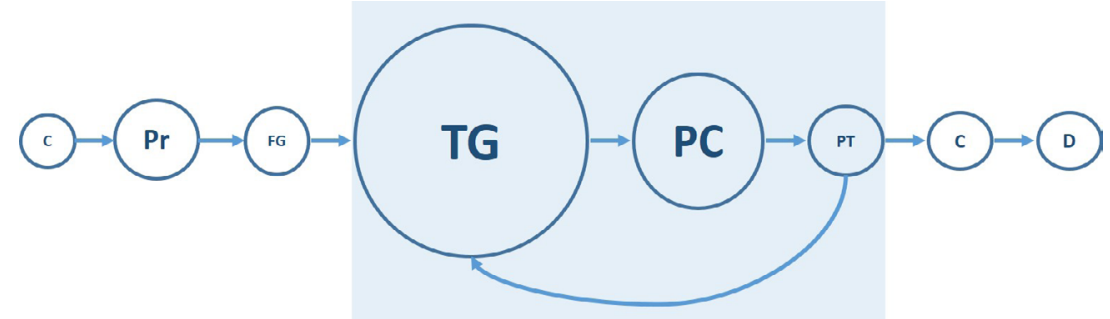

Figura 2. Modelo metodológico posible

El ciclo se inicia con el cuestionario de conocimientos previos (C), seguido de la presentación del tema y la metodología a emplear (Pr) y la formación de los grupos de trabajo (FG). Las actividades centrales seguirán el esquema:

- TG: Trabajo en grupo, en diferentes formatos: búsqueda de información, tratamiento de datos, representaciones gráficas... Interacción con la profesora que acompaña a los grupos a ratos, resuelve dudas puntuales y proporciona retroalimentación.

- PC: Puesta en común de los resultados del trabajo en grupo. Retroalimentación de los compañeros y de la profesora.

- PT: Presentación por parte de la profesora de un resumen de los contenidos que se han abordado en la actividad y resolución de dudas.

Al final de la última sesión del ciclo de mejora, las dos últimas actividades serán el cuestionario final (C) y la despedida y valoración de las sesiones (D).

Para desarrollar los contenidos seleccionados y en aplicación de este modelo metodológico, se ha optado por 
actividades cortas que trabajen en grupo contenidos muy concretos, seguidas de puestas en común y, en determinados casos, resúmenes de los contenidos por parte de la profesora, con la función, en ambos casos, de actividades de contraste (de Alba y Porlán, 2017). Como resultado, para el ciclo de mejora completo se han preparado 28 actividades, que no se pueden presentar aquí por falta de espacio. Cada una de las cuatro sesiones inicialmente programadas estaba dedicada a uno de los grandes bloques de contenidos, representado por un problema, tal como aparece en la Tabla 1.

Tabla 1. Contenidos y problemas tratados en las cuatro sesiones previstas

\begin{tabular}{|c|c|c|}
\hline Sesión & Contenido & Pregunta/problema \\
\hline 1 & $\begin{array}{c}\text { Las fuentes y el método } \\
\text { estadístico }\end{array}$ & $\begin{array}{c}\text { ¿Qué es y para qué sirve el baró- } \\
\text { metro del CIS? ¿A quién hace sus } \\
\text { encuestas? }\end{array}$ \\
\hline 2 & $\begin{array}{c}\text { Variables estadísti- } \\
\text { cas. Tipos y niveles de } \\
\text { medición. } \\
\text { Tablas de frecuencias }\end{array}$ & ¿Qué preguntan las encuestas del \\
ClS?
\end{tabular}

Como ejemplo, en la Tabla 2 se presenta la secuencia de actividades prevista para la sesión número 4, en la que se trataba el tema de la ética estadística.

Jornadas de Formación e Innovación Docente del Profesorado | № 2 (2019) Esta obra se distribuye con la licencia Creative Commons Reconocimiento-NoComercial-SinObraDerivada Internacional (CC BY-NC-ND 4.0.) 
Tabla 2. Secuencia de actividades prevista para la sesión 4.

\begin{tabular}{|c|c|c|c|}
\hline $\begin{array}{l}\text { Descripción de la } \\
\text { actividad (Tiempo) }\end{array}$ & Finalidad & $\begin{array}{c}\text { Papel de alumnos } \\
\text { y profesora }\end{array}$ & Contenidos \\
\hline $\begin{array}{l}\text { 23. Analizar los re- } \\
\text { sultados sobre } \\
\text { intención de voto pu- } \\
\text { blicados por el baró- } \\
\text { metro de febrero de } \\
2019 \text { y compararlos } \\
\text { con los publicados } \\
\text { en enero de } 2018 \text {, po- } \\
\text { niéndolos en relación } \\
\text { con la metodología } \\
\text { empleada ( } 30 \mathrm{~min} \text { ) }\end{array}$ & \multirow{2}{*}{$\begin{array}{l}\text { Tomar con- } \\
\text { ciencia de } \\
\text { la importan- } \\
\text { cia del trata- } \\
\text { miento de los } \\
\text { datos estadís- } \\
\text { ticos y sus im- } \\
\text { plicaciones } \\
\text { éticas. }\end{array}$} & $\begin{array}{l}\text { Estudiantes traba- } \\
\text { jan y discuten por } \\
\text { grupos. Profesora } \\
\text { acompaña, escucha } \\
\text { y se integra en los } \\
\text { grupos. }\end{array}$ & \multirow{4}{*}{$\begin{array}{l}\text { ¿Es neu- } \\
\text { tral el tra- } \\
\text { tamiento } \\
\text { que se da } \\
\text { a los da- } \\
\text { tos? La ética } \\
\text { estadística }\end{array}$} \\
\hline $\begin{array}{l}\text { 24. Poner en común } \\
\text { los resultados de la } \\
\text { actividad } 23 \text { ( } 15 \text { min) }\end{array}$ & & $\begin{array}{l}\text { Grupos exponen } \\
\text { sus conclusiones } \\
\text { sobre la Actividad } \\
\text { 23. Otros grupos } \\
\text { dan su opinión y se } \\
\text { debate. }\end{array}$ & \\
\hline $\begin{array}{l}\text { 25. Examinar ejem- } \\
\text { plos de manipulación } \\
\text { a través de la publi- } \\
\text { cación de tablas o } \\
\text { gráficos estadísticos } \\
\text { (30 min) }\end{array}$ & \multirow{2}{*}{$\begin{array}{l}\text { Tomar con- } \\
\text { ciencia de los } \\
\text { posibles ma- } \\
\text { los usos de } \\
\text { la estadística, } \\
\text { intencionados } \\
\text { o no }\end{array}$} & $\begin{array}{l}\text { Estudiantes traba- } \\
\text { jan y discuten por } \\
\text { grupos. Profesora } \\
\text { acompaña, escucha } \\
\text { y se integra en los } \\
\text { grupos. }\end{array}$ & \\
\hline $\begin{array}{l}\text { 26. Poner en común } \\
\text { los resultados de la } \\
\text { actividad } 25 \text { ( } 15 \mathrm{~min})\end{array}$ & & $\begin{array}{l}\text { Grupos exponen } \\
\text { sus conclusiones } \\
\text { sobre la Actividad } \\
\text { 25. Otros grupos } \\
\text { dan su opinión y se } \\
\text { debate. }\end{array}$ & \\
\hline
\end{tabular}

Jornadas de Formación e Innovación Docente del Profesorado I № 2 (2019) Esta obra se distribuye con la licencia Creative Commons 


\begin{tabular}{|l|l|l|l|}
\hline $\begin{array}{l}\text { 27. Pasar el cuestio- } \\
\text { nario final (20 min) }\end{array}$ & $\begin{array}{l}\text { Evaluar los } \\
\text { conocimien- } \\
\text { tos adquiridos } \\
\text { y proporcio- } \\
\text { nar infor- } \\
\text { mación para } \\
\text { construir las } \\
\text { escaleras de } \\
\text { aprendizaje }\end{array}$ & $\begin{array}{l}\text { Estudiantes res- } \\
\text { ponden al cues- } \\
\text { tionario, profesora } \\
\text { acompaña, observa } \\
\text { y anota. }\end{array}$ & \\
\hline $\begin{array}{l}\text { 28. Valoración de la } \\
\text { metodología y de las } \\
\text { actividades del CIMA } \\
\text { (10 min) }\end{array}$ & $\begin{array}{l}\text { Conocer la } \\
\text { opinión de } \\
\text { los estudian- } \\
\text { tes sobre el } \\
\text { desarrollo } \\
\text { de las sesio- } \\
\text { nes del Cima } \\
\text { y su percep- } \\
\text { ción sobre su } \\
\text { aprendizaje }\end{array}$ & \\
\hline
\end{tabular}

\section{Cuestionario inicial-final}

Para construir el aprendizaje es necesario partir de las ideas previas o los esquemas mentales de los estudiantes (Rivero y Porlán, 2017). Una forma de identificarlos es utilizar un cuestionario sobre los contenidos que se van a realizar y que los estudiantes deben responder antes de iniciar las sesiones. Además de proporcionar información a la profesora sobre los modelos mentales de partida del grupo de estudiantes, este cuestionario cumple la función de hacerles a ellos conscientes de lo que ya saben sobre el tema y de lo que desconocen, lo que les pone en posición de aprender mejor. El mismo cuestionario se utiliza, al finalizar las sesiones, para evaluar el grado de aprendizaje de los contenidos trabajados.

El cuestionario diseñado para este fin se compone de las cinco preguntas siguientes: 
1. A partir de las convocatorias de elecciones es habitual que en los medios de comunicación se hable del CIS (Centro de Investigaciones Sociológicas), ¿sabes a qué se dedica este organismo? ¿Qué son los Barómetros del CIS? ¿Para qué sirven?

2. Imagina que necesitas conocer la opinión de la sociedad española en su conjunto sobre algún tema y, claro está, no puedes acceder a todos los habitantes del país, ¿sería posible seleccionar a un grupo de personas representativas del conjunto de la sociedad? ¿Cómo lo harías? Justifica tu respuesta.

3. En un grupo de 10 estudiantes del Grado en Geografia y Gestión del Territorio se ha observado el conjunto de variables que aparece en la tabla siguiente (provincia de nacimiento, cociente intelectual, color de ojos, peso, estatura y talla de camiseta). Clasifica las variables en función de lo que permitan hacer: clasificar a los individuos, ordenar a los individuos y calcular diferencias (absolutas y/o relativas) entre ellos.

\begin{tabular}{|c|c|c|c|c|c|c|}
\hline Estudiante & $\begin{array}{c}\text { Provincia de } \\
\text { nacimiento }\end{array}$ & $\begin{array}{c}\text { Cociente } \\
\text { intelectual }\end{array}$ & $\begin{array}{c}\text { Color } \\
\text { de ojos }\end{array}$ & $\begin{array}{c}\text { Peso } \\
(\mathrm{kg})\end{array}$ & $\begin{array}{c}\text { Estatura } \\
(\mathrm{cm})\end{array}$ & $\begin{array}{c}\text { Talla de } \\
\text { camiseta }\end{array}$ \\
\hline A & Sevilla & 100 & Marrón & 65.5 & 171.5 & $\mathrm{M}$ \\
\hline B & Málaga & 101 & Azul & 89.8 & 187.2 & $\mathrm{XL}$ \\
\hline C & Cádiz & 94 & Marrón & 54.1 & 165.7 & $\mathrm{~S}$ \\
\hline D & Sevilla & 102 & Negro & 78.9 & 160.9 & $\mathrm{~L}$ \\
\hline E & Sevilla & 99 & Marrón & 68.1 & 175.4 & $\mathrm{~L}$ \\
\hline F & Sevilla & 105 & Marrón & 52.7 & 158.9 & $\mathrm{XS}$ \\
\hline G & Badajoz & 89 & Verde & 95.6 & 193.5 & $\mathrm{XXL}$ \\
\hline H & Cádiz & 91 & Negro & 67.2 & 174.6 & $\mathrm{XL}$ \\
\hline I & Sevilla & 114 & Marrón & 65.9 & 169.5 & $\mathrm{~L}$ \\
\hline J & Huelva & 147 & Marrón & 60.3 & 175.4 & $\mathrm{M}$ \\
\hline
\end{tabular}


4. Una ojeadora de baloncesto quiere representar gráficamente el número de puntos anotados por una serie de jugadores en un torneo. Las puntuaciones son 86, 88, 90, 90, 97, 99. 102 y 106. ¿Cuál de los siguientes gráficos ( $a, b, c, d$ o e) crees que es el más correcto para representar esos datos? ¿Por qué?
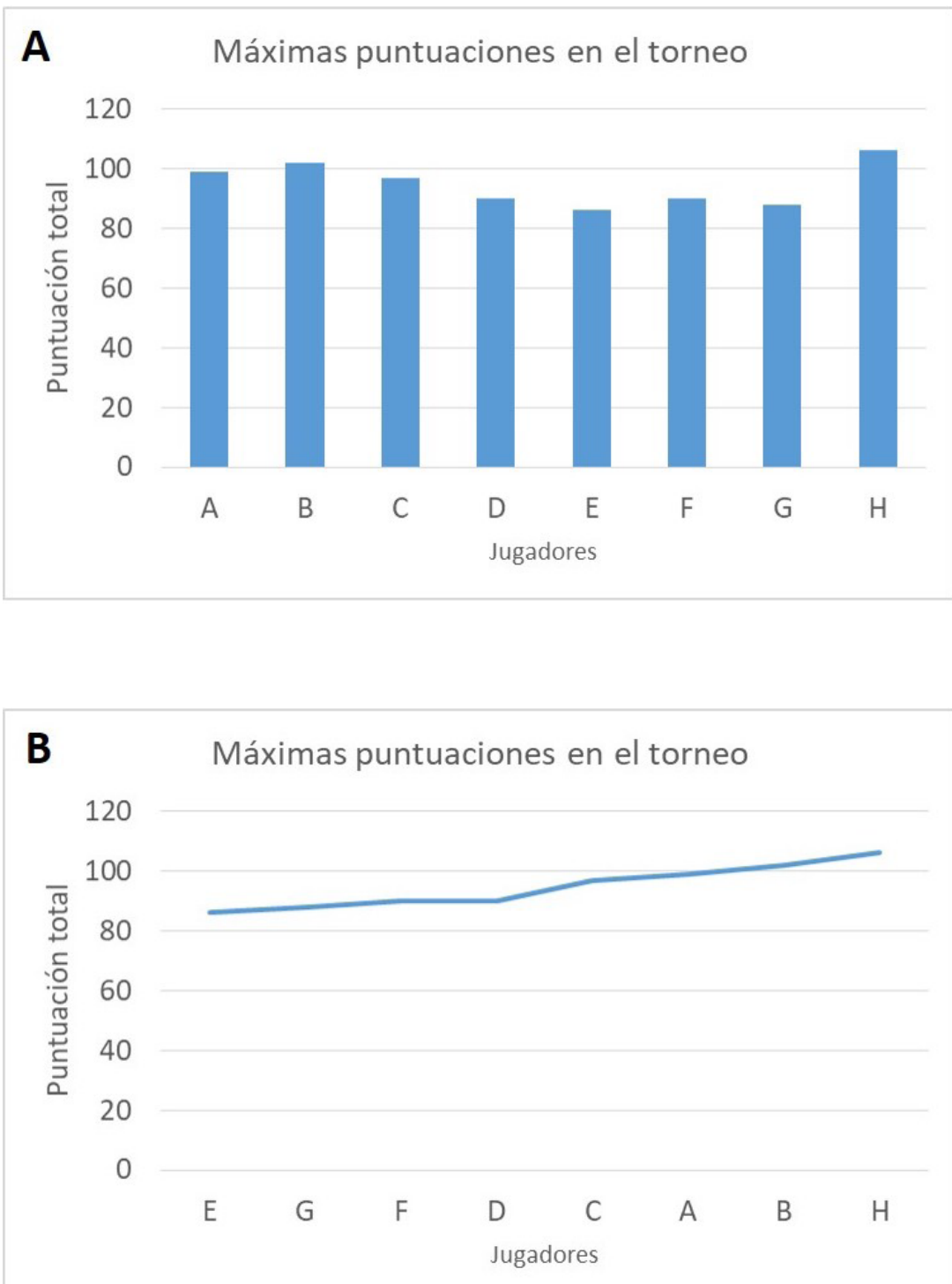

Jornadas de Formación e Innovación Docente del Profesorado | № 2 (2019) Esta obra se distribuye con la licencia Creative Commons Reconocimiento-NoComercial-SinObraDerivada 4.0 Internacional (CC BY-NC-ND 4.0.) 


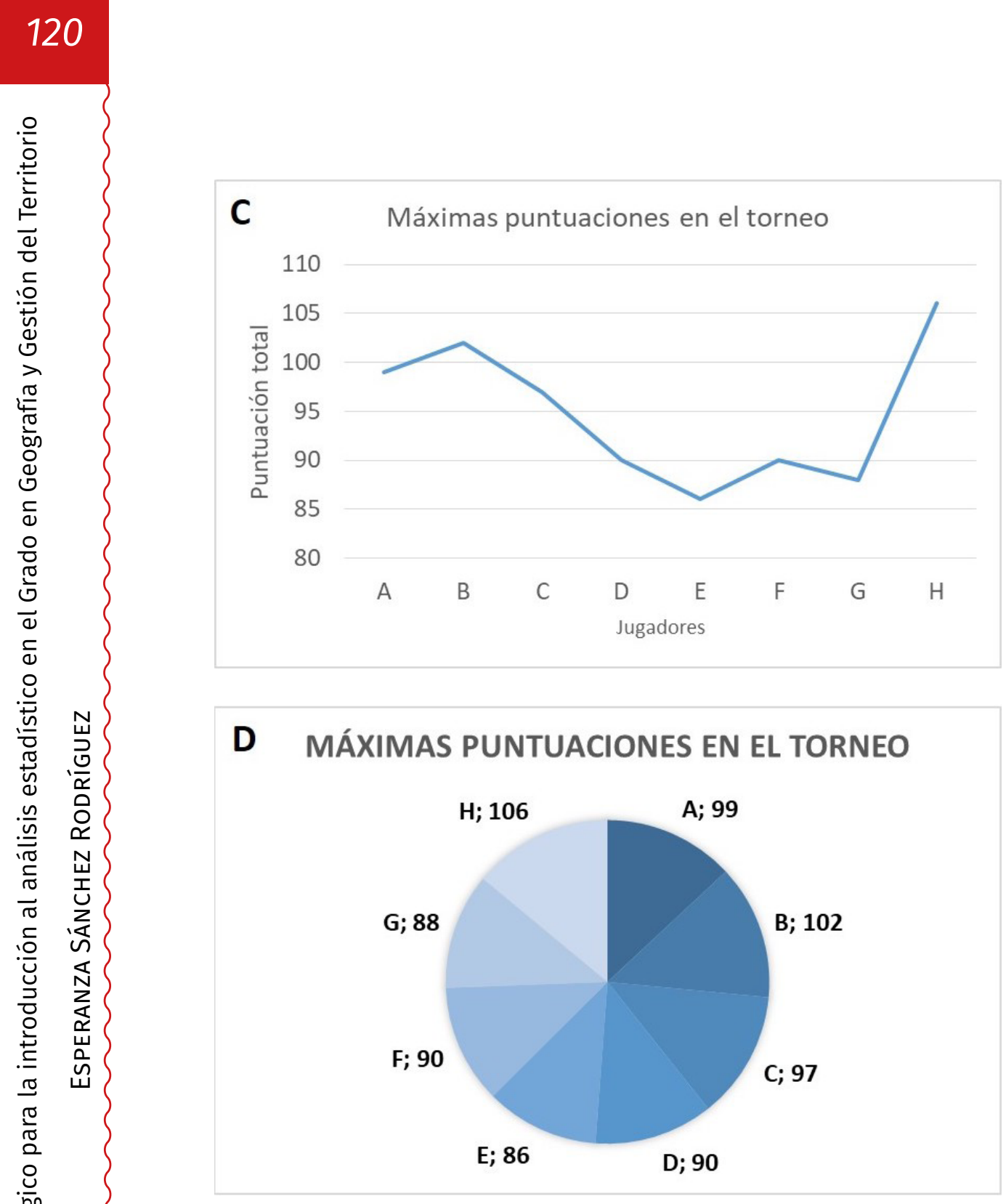

(Figura 4)

5. A partir de la observación del índice de masa corporal en 40 hombres hemos realizado las siguientes tablas. Describe cómo es el IMC en el grupo estudiado y elige la tabla ( $a, b$ o c) que consideras más adecuada para describir esta variable. Justifica tu respuesta.

Jornadas de Formación e Innovación Docente del Profesorado I № 2 (2019) Esta obra se distribuye con la licencia Creative Commons Reconocimiento-NoComercial-SinObraDerivada 4.0 Internacional (CC BY-NC-ND 4.0.) 


\begin{tabular}{|c|c|c|c|c|c|}
\hline \multicolumn{2}{|c|}{ A } & \multicolumn{2}{c|}{ B } & \multicolumn{2}{c|}{ C } \\
\hline Clase & Frecuencia & Clase & Frecuencia & Clase & Frecuencia \\
\hline $15-20.2$ & 9 & $<20$ & 8 & $15-19.9$ & 8 \\
$20.3-25.3$ & 13 & $20-25$ & 10 & $20-24.9$ & 10 \\
$25.4-28.7$ & 8 & $25-30$ & 15 & $25-29.9$ & 15 \\
$28.8-32.1$ & 5 & $30-35$ & 4 & $30-34.9$ & 4 \\
$32.2-35.5$ & 2 & $35-40$ & 1 & $35-39.9$ & 1 \\
$35.6-42.8$ & 2 & $>40$ & 2 & $40-44.9$ & 2 \\
$42.9-45$ & 1 & & & & \\
\hline
\end{tabular}

La reflexión posterior ha permitido detectar algunas cuestiones mejorables en el cuestionario, como, por ejemplo, el hecho de que no incluye ninguna pregunta sobre uno de los bloques de contenidos, el de la ética estadística.

\section{Aplicación del ciclo de mejora en el aula}

\section{Relato resumido de las sesiones}

Inicié las clases del segundo ciclo de mejora el día 4 de abril con mucha ilusión y un poco nerviosa. Me hacía ilusión cambiar la forma de trabajar con los alumnos y, por otro lado, había trabajado mucho en la preparación de las actividades y estaba deseando ver si funcionaban. A esta primera sesión del CIMA asistieron 12 de los 16 alumnos.

Tras pasar el cuestionario inicial, presenté el tema a tratar (ayudándome del mapa de contenidos, que atrajo la atención de los alumnos bastante más que si hubiera sido una lista al modo convencional), la metodología que ibamos a usar en estas sesiones y les expliqué el sistema de calificación que proponía. Les hice saber que la aplicación de esta metodología requiere un compromiso por su parte y su participación activa. Finalmente, les pregunté su opinión sobre la evaluación y la metodología y si estaban dispuestos a comprometerse. La respuesta fue afirmativa; 
todos contestaron expresamente que sí se comprometían a trabajar y que les parecía adecuada la forma de calificar su participación.

Tras formar los grupos (de tres, para que pudieran comunicarse bien en una clase con las mesas fijas), empezamos a trabajar en los contenidos con la Actividad 4 que consistía en investigar sobre el CIS y el barómetro, respondiendo a una serie de preguntas que les proporcioné por escrito. Se dedicaron a ello con entusiasmo y mucho interés. Los diferentes grupos adoptaron distintas estrategias: algunos se dividieron las preguntas y cada uno buscó las respuestas a las suyas; en otros grupos, desde el principio se plantearon hacerlo todo juntos, e iban respondiendo a cada pregunta, con uno de ellos haciendo de secretario y tomando nota. Evidentemente, los grupos que se dividieron el trabajo terminaron antes, aunque yo creo que los que trabajan a la vez aprenden más. Me llamó la atención que trabajaron de forma muy autónoma y con mucho interés. Yo había previsto para esta actividad unos 20 minutos y tardaron el doble. Eso me causó cierto nerviosismo y mientras ellos trabajaban revisé varias veces la secuencia de actividades para ver de dónde podría recortar un poco de tiempo.

En la puesta en común de este tema participaron todos. Pedía a un grupo que contestara a una pregunta y los otros comentaban después si habían encontrado más información o la habían enfocado de otra forma. En esta actividad me di cuenta de que es más dificil de lo que parece dirigir una puesta en común. Si no estaba muy atenta, mi tendencia era a hablar yo (especialmente porque había planificado poco tiempo para la puesta en común, debido a mi inexperiencia en este tipo de actividades), y la de ellos a dejarme hacerlo; es dificil cambiar hábitos que se han practicado durante mucho tiempo. Este ha sido uno de mis problemas en la aplicación de este 2 CIMA. Además, debo dejar más tiempo para todas las actividades que 
supongan exposición por parte de los alumnos, porque ellos tienen otro ritmo diferente y necesitan su tiempo. El resto de la sesión se desarrolló siguiendo la misma tónica: trabajo en grupo con mucho interés por parte de los alumnos y puestas en común. De nuevo me quedé corta en la estimación del tiempo en mi previsión.

La segunda sesión tuvo lugar el día 10 de abril. En esta ocasión asistieron 15 alumnos. En general, durante esta sesión las actividades funcionaron. Alternamos trabajo en equipo, puestas en común y de vez en cuando un resumen por parte de la profesora. En esta sesión el trabajo en equipo se centra en el manejo de datos concretos. Esto les descoloca un poco más, les crea más incertidumbre y están un poco más inseguros, lo que se traduce en que preguntan más. Sin embargo, lo solucionan muy bien aunque les cuesta explicar por qué han tomado decisiones o aplicado métodos. Me doy cuenta de que les interesa trabajar con datos reales, no inventados ni reducidos para las actividades de clase, como ya se ha comprobado en muchas ocasiones (Batanero y Díaz, 2004). Les motiva y les incentiva saber que están aplicando de verdad las diferentes fases de la investigación estadística y que esos mismos pasos son los que han dado los especialistas del CIS en el análisis de estos datos.

El principal problema sigue siendo que he fijado demasiado poco tiempo para cada actividad. Seguimos acumulando retraso sobre el horario previsto y me pongo nerviosa. Sobre la marcha, elimino uno o dos apartados de alguna actividad para intentar recuperar un poco de tiempo. Los alumnos notan mi nerviosismo y me preguntan; cuando les digo que he planificado las actividades para hacerlas en menos tiempo, no se preocupan; me dicen que dediquemos más clases a este tema y ya está. En ese momento, ya me rindo y me resigno a alargar el tema una clase más, dedicando al 2CIMA 10 horas en lugar de 8. Desde entonces, la clase va mucho mejor y yo la disfruto más. 
En la tercera sesión, realizada el día 11 de abril, asisten 14 alumnos. La tónica sigue siendo la misma: los alumnos trabajan actividades prácticas sobre los datos del barómetro de febrero por grupos, se ponen en común los resultados y finalmente si es necesario la profesora resume los contenidos trabajados y muestra nuevas posibilidades de análisis que no hayan aparecido. Siguen trabajando con interés y en estas actividades más concretas que trabajan ya con datos específicos y piden un resultado concreto las diferencias entre los grupos son menores. Siguen tardando más de lo que yo tenía previsto, pero ya lo he aceptado y estoy tranquila, disfrutando la clase.

La cuarta sesión del segundo ciclo de mejora se realizó el día 24 de abril. En esta sesión tratábamos el tema de los gráficos estadísticos y pensaba que les interesarían, aunque me quedaba la duda de cómo relacionarían los conceptos con los ya vistos, debido al parón de las vacaciones de Semana Santa. Por otra parte, consideraba fundamental que esta sesión funcionara bien, ya que, si bien la construcción y la interpretación correcta de gráficos es una habilidad básica para un geógrafo e incluso para cualquier ciudadano, esta capacidad a menudo no alcanza el nivel suficiente entre los alumnos universitarios (Arteaga, Batanero y Cañadas, 2010; Hernández, García y López, 2019). En esta ocasión asistieron 14 alumnos. El desarrollo de la sesión siguió la misma tónica que en las anteriores; estudiantes motivados y trabajando. En este caso fue aún más ágil y entretenido, ya que estuvieron haciendo gráficos con Excel y hubo mucho intercambio de ideas sobre diseño, selección de tipos de gráficos, etc. La puesta en común fue muy interesante: ellos me enviaron sus gráficos por correo electrónico y yo los proyecté en la pantalla. Opinaban sobre los gráficos de otros grupos y fue bastante dinámico. De todas formas, creo que sigo hablando demasiado en este tipo de actividades de contraste. Mi intención es solamente resumir y ordenar las ideas expuestas, pero siempre acabo hablando más de lo que quiero; quizá 
sea también una cuestión de la disposición de la clase (todos mirando en la misma dirección, de manera que para que me vean tengo que ponerme delante de ellos, en la posición que ocupa el profesor en las clases expositivas tradicionales). Acabé la clase muy contenta por la participación y actitud de los alumnos. Me divertí y me pareció que aprendían.

La última sesión del segundo ciclo de mejora tuvo lugar el jueves 25 de abril. Es la sesión que más ilusión me hacía ya que íbamos a tratar el tema de la ética estadística, que nunca antes había tratado en clase. Tenía mucha curiosidad por la reacción de los alumnos y el interés que el tema despertaría en ellos. A esta sesión asistieron sólo 10 alumnos. Algunos de los que faltaron me habían avisado el día anterior.

El tema les interesó muchísimo. La primera actividad fue sobre la "cocina" del Barómetro del CIS, el tratamiento que se da a los datos de intención de voto, que se ha cambiado desde la incorporación del último director del CIS. El trabajo en grupo fue muy animado, se les veía leer y comentar los materiales con mucha implicación. Todos querían dar su opinión a la hora de la puesta en común. A ello contribuyó, por supuesto, la fecha de la sesión a sólo 3 días de las elecciones generales del 28 de abril. También participaron activamente en las demás actividades. Disfruté mucho la sesión, me divertí y aprendí de las aportaciones de los estudiantes. Verlos motivados me cargó las pilas. Durante toda la sesión se vivió un ambiente de trabajo e intercambio de ideas muy interesante. Terminamos la sesión con el cuestionario final y el cuestionario de evaluación de la metodología. 


\section{Evaluación del aprendizaje de los estudiantes}

El cuestionario se utilizó también al finalizar las sesiones del segundo ciclo de mejora para evaluar los cambios en los modelos mentales del alumnado y los conocimientos conceptuales y procedimentales adquiridos durante las sesiones. Fue respondido en clase por 10 estudiantes.

El examen de las soluciones que los estudiantes dieron al cuestionario permitió reconocer diferentes modelos de respuestas que se muestran en la Tabla 3, y éstos a su vez posibilitaron la identificación de los principales obstáculos en el aprendizaje de los contenidos tratados en cada cuestión, lo que considero información de gran utilidad para la docencia de la asignatura en el futuro, puesto que su identificación permitirá el diseño de actividades específicas para superarlos.

A partir de esos modelos, se asigna a cada estudiante el nivel correspondiente en cada una de las preguntas del cuestionario inicial y final. El resultado para los 10 alumnos que completaron ambos cuestionarios se muestra en la Tabla 4. En general se observa un progreso importante en el nivel de los estudiantes; 9 de los 10 estudiantes han mejorado su nivel al menos en cuatro de las cinco cuestiones planteadas y en tres de los casos en que el estudiante no ha mejorado su nivel se ha debido a que ya se encontraba en el escalón más alto y no existía posibilidad de mejora. Hay que señalar, sin embargo, que un estudiante (4) parece haber retrocedido en su nivel de conocimientos en una de las preguntas (la cuarta); sin embargo, en las otras preguntas su nivel ha mejorado significativamente. 
Tabla 3. Definición de los modelos de respuestas

\begin{tabular}{|c|c|c|c|c|c|}
\hline & Pregunta 1 & Pregunta 2 & Pregunta 3 & Pregunta 4 & Pregunta 5 \\
\hline$D$ & $\begin{array}{l}\text { No contesta o } \\
\text { sólo lo identi- } \\
\text { fica como orga- } \\
\text { nismo público }\end{array}$ & $\begin{array}{l}\text { Concepto de } \\
\text { muestra }\end{array}$ & $\begin{array}{l}\text { Diferen- } \\
\text { cia variables } \\
\text { cuantitativas } \\
\text { de cualita- } \\
\text { tivas }\end{array}$ & $\begin{array}{l}\text { Elige el gráfico } \\
\text { D, no aplicable } \\
\text { porque de esta } \\
\text { variable no in- } \\
\text { teresa el total }\end{array}$ & $\begin{array}{l}\text { Elige la tabla A, } \\
\text { (intervalos de } \\
\text { diferente ampli- } \\
\text { tud) y no des- } \\
\text { cribe la variable }\end{array}$ \\
\hline$C$ & $\begin{array}{l}\text { Sabe que el CIS } \\
\text { estudia cuestio- } \\
\text { nes sociológicas } \\
\text { y preferencias } \\
\text { políticas }\end{array}$ & $\begin{array}{l}\text { Aleatorie- } \\
\text { dad de la } \\
\text { muestra }\end{array}$ & $\begin{array}{l}\text { Identifica al } \\
\text { menos el ni- } \\
\text { vel nominal }\end{array}$ & $\begin{array}{l}\text { Elige los gráfi- } \\
\text { cos B o C (no } \\
\text { adecuados ya } \\
\text { que las obser- } \\
\text { vaciones son } \\
\text { independien- } \\
\text { tes) }\end{array}$ & $\begin{array}{l}\text { Elige la tabla B, } \\
\text { (intervalos que } \\
\text { se solapan) y } \\
\text { no describe la } \\
\text { variable }\end{array}$ \\
\hline B & $\begin{array}{l}\text { Es una fuente } \\
\text { estadística }\end{array}$ & $\begin{array}{l}\text { División de } \\
\text { la población } \\
\text { en estratos }\end{array}$ & $\begin{array}{l}\text { Identifica al } \\
\text { menos el ni- } \\
\text { vel ordinal }\end{array}$ & $\begin{array}{l}\text { Elige el gráfico } \\
\text { adecuado (A) }\end{array}$ & $\begin{array}{l}\text { Elige la tabla B, } \\
\text { pero describe la } \\
\text { variable o elige } \\
\text { la C pero no } \\
\text { describe }\end{array}$ \\
\hline$A$ & $\begin{array}{l}\text { Estudia mues- } \\
\text { tras para re- } \\
\text { presentar la } \\
\text { población }\end{array}$ & $\begin{array}{l}\text { Necesidad } \\
\text { de un ta- } \\
\text { maño mues- } \\
\text { tral mínimo }\end{array}$ & $\begin{array}{l}\text { Identifica co- } \\
\text { rrectamente } \\
\text { los nive- } \\
\text { les de las } \\
\text { variables }\end{array}$ & $\begin{array}{l}\text { Elige el grá- } \\
\text { fico adecuado } \\
\text { (A) y sabe } \\
\text { justificarlo }\end{array}$ & $\begin{array}{l}\text { Elige la tabla } \\
\text { C y describe la } \\
\text { variable. }\end{array}$ \\
\hline
\end{tabular}

Tabla 4. Seguimiento de cada estudiante

\begin{tabular}{|c|c|c|c|c|c|c|c|c|c|c|}
\hline \multirow[b]{2}{*}{ Estudiante } & \multicolumn{2}{|c|}{ Pregunta 1} & \multicolumn{2}{|c|}{ Pregunta 2} & \multicolumn{2}{|c|}{ Pregunta 3} & \multicolumn{2}{|c|}{ Pregunta 4} & \multicolumn{2}{|c|}{ Pregunta 5} \\
\hline & $\begin{array}{l}\frac{\vec{U}}{U} \\
\underline{\underline{I}}\end{array}$ & 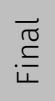 & $\stackrel{\overrightarrow{\underline{U}}}{. \underline{\underline{\underline{U}}}}$ & $\begin{array}{l}\stackrel{\tilde{\sigma}}{\leftrightarrows} \\
\dot{\leftarrow}\end{array}$ & $\begin{array}{l}\frac{\vec{v}}{\underline{U}} \\
\underline{\underline{E}}\end{array}$ & 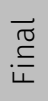 & $\begin{array}{l}\frac{\vec{U}}{\underline{U}} \\
\underline{\underline{I}}\end{array}$ & 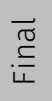 & . $\frac{\overrightarrow{\underline{U}}}{\underline{\underline{\underline{U}}}}$ & 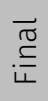 \\
\hline 1 & C & $\mathrm{B}$ & B & B & D & C & $D$ & B & C & C \\
\hline 2 & B & $A$ & B & A & $D$ & B & B & $A$ & $D$ & C \\
\hline 3 & B & $A$ & $C$ & B & $D$ & B & $A$ & $A$ & C & B \\
\hline 4 & C & $A$ & $D$ & C & $B$ & $A$ & B & $C$ & $D$ & C \\
\hline 5 & C & $A$ & $\mathrm{D}$ & B & $\mathrm{D}$ & B & $D$ & $C$ & $C$ & B \\
\hline
\end{tabular}




\begin{tabular}{|c|c|c|c|c|c|c|c|c|c|c|}
\hline 6 & $\mathrm{D}$ & $\mathrm{C}$ & $\mathrm{B}$ & $\mathrm{B}$ & $\mathrm{D}$ & $\mathrm{B}$ & $\mathrm{B}$ & $\mathrm{A}$ & $\mathrm{D}$ & $\mathrm{A}$ \\
\hline 7 & $\mathrm{C}$ & $\mathrm{A}$ & $\mathrm{C}$ & $\mathrm{A}$ & $\mathrm{D}$ & $\mathrm{B}$ & $\mathrm{B}$ & $\mathrm{A}$ & $\mathrm{A}$ & $\mathrm{A}$ \\
\hline 8 & $\mathrm{~B}$ & $\mathrm{~A}$ & $\mathrm{C}$ & $\mathrm{A}$ & $\mathrm{B}$ & $\mathrm{A}$ & $\mathrm{C}$ & $\mathrm{C}$ & $\mathrm{D}$ & $\mathrm{C}$ \\
\hline 9 & $\mathrm{~B}$ & $\mathrm{~A}$ & $\mathrm{C}$ & $\mathrm{A}$ & $\mathrm{D}$ & $\mathrm{B}$ & $\mathrm{C}$ & $\mathrm{B}$ & $\mathrm{D}$ & $\mathrm{B}$ \\
\hline 10 & $\mathrm{~A}$ & $\mathrm{~A}$ & $\mathrm{D}$ & $\mathrm{C}$ & $\mathrm{D}$ & $\mathrm{B}$ & $\mathrm{B}$ & $\mathrm{A}$ & $\mathrm{D}$ & $\mathrm{B}$ \\
\hline
\end{tabular}

Los resultados también se han representado en forma de escaleras de aprendizaje para cada pregunta. Como ejemplo, se presenta en la Figura 3 la realizada para la pregunta 2; en ella aparece el porcentaje de estudiantes en cada nivel en el cuestionario inicial (izquierda) y final (derecha), así como los principales obstáculos identificados gracias al análisis de sus respuestas, que fueron tres. El primero, que es necesario salvar para acceder al nivel C hace referencia a la intervención del azar en el proceso de selección de la muestra para evitar su sesgo. El segundo obstáculo se encuentra entre el nivel C y el B y se refiere a tener conciencia de que en la muestra deben estar representados los diversos sectores económicos, sociales, y los diferentes territorios de España para que realmente represente a toda la sociedad, lo que se resumen en el concepto de muestreo estratificado. Finalmente, el obstáculo que se encuentra entre el nivel $B$ y el $A$ hace referencia a ser consciente de que es necesario un tamaño mínimo para que una muestra represente realmente a una población.

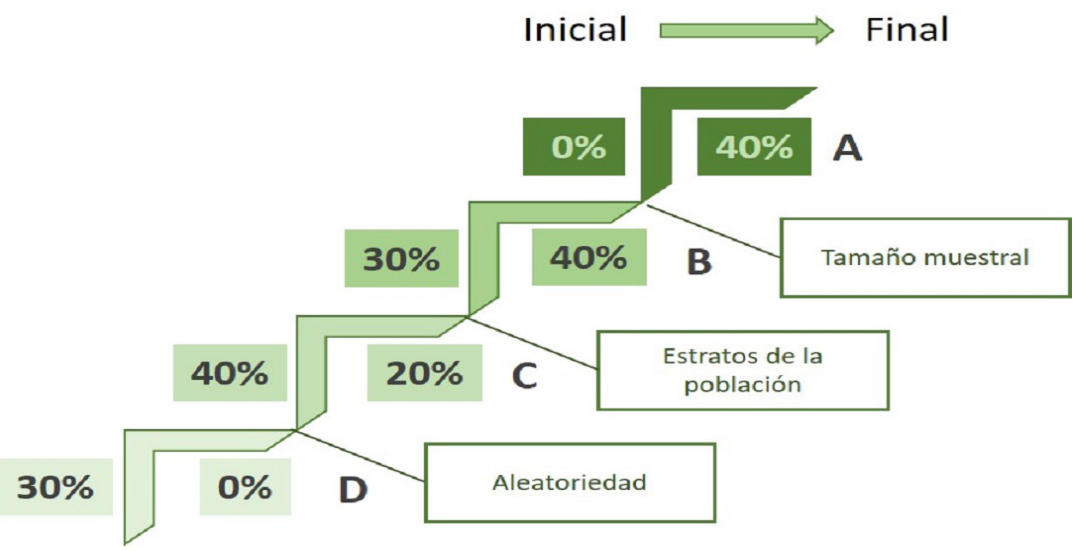

Figura 3. Escalera de aprendizaje para la pregunta 2

Jornadas de Formación e Innovación Docente del Profesorado | № 2 (2019) Esta obra se distribuye con la licencia Creative Commons Reconocimiento-NoComercial-SinObraDerivada Internacional (CC BY-NC-ND 4.0.) 


\section{Evaluación del ciclo de mejora en el aula}

El trabajo en el CGDU me ha hecho consciente de que en cualquier clase estamos aplicando una determinada metodología, un modelo didáctico sea consciente o no (García y Porlán, 2017); es decir, que si elijo hacer una clase expositiva siguiendo el modelo tradicional transmisivo ya estoy haciendo una elección metodológica. No quiero seguir dando todas las clases con ese modelo metodológico, que ha demostrado no ser demasiado útil. Aunque no tengo definido un modelo metodológico ideal único, sí tengo claro que en los modelos metodológicos posibles que aplicaré en mis clases tendré en cuenta:

- Empezar cada tema con el planteamiento de un problema que pueda ser de interés para los alumnos y que sirva de ejemplo de la utilidad o interés de los contenidos que vamos a tratar.

- En todas las clases plantear a los estudiantes alguna actividad intelectual que vaya más allá de escuchar y anotar (Finkel, 2008), que tengan que producir algún resultado, preferentemente escrito. Ello les ayudará a concretar sus ideas, a cuestionárselas y a razonar.

- Incorporar el trabajo en grupo en muchas de las clases, ya que he comprobado en los dos ciclos de mejora que puede dar muy buenos resultados, motivando a los estudiantes y estimulándolos intelectualmente.

- Utilizar secuencias de actividades variadas, que no sigan siempre el mismo modelo (cambiando el tipo de actividades o el orden), para evitar que los alumnos se acomoden demasiado y mantener un cierto nivel de tensión intelectual.

- Al final de cada sesión pedir que resuman qué contenidos se han trabajado, para hacerlos conscientes del proceso de construcción de su aprendizaje.

- Terminar cada tema con una pregunta que puedan considerar interesante, y no necesariamente contestarla (Bain, 2007). Darles la oportunidad de probar 
 \\ lo que han aprendido dejándoles una cuestión en la que aplicarlo, si lo desean. \\ - Pedir siempre a los alumnos compromiso con su proceso de aprendizaje, con el desarrollo de la clase (Bain, 2007). Vincular ese compromiso, al menos al principio, con la calificación de alguna forma. \\ La realización del Curso General de Docencia Univer- sitaria y la puesta en práctica de los dos ciclos de mejora que ello implica me ha proporcionado algunas herramien- tas y, sobre todo, la motivación para iniciar un cambio progresivo en mi docencia que permita una mayor impli- cación del alumnado en su proceso de aprendizaje, de ma- nera que aprendan más y mejor.}

Jornadas de Formación e Innovación Docente del Profesorado | № 2 (2019) Esta obra se distribuye con la licencia Creative Commons 


\section{Referencias biliográficas}

Arteaga, P., Batanero, C. y Cañadas, G. (2010). Gráficos estadisticos en la formación de profesores. En J. Ortiz (Ed.). Investigaciones actuales en educación estadística y formación de profesores (pp. 73-88). Granada: Universidad de Granada.

Bain, K. (2007). Lo que hacen los mejores profesores universitarios. Valencia: Universitat de Valencia.

Batanero, C. y Díaz, C. (2004). El Papel de los Proyectos en la Enseñanza y Aprendizaje de la Estadística. En P. Royo (Ed.). Aspectos didácticos de las matemáticas (pp. 125164). Zaragoza: ICE.

De Alba, N. y Porlán, R. (2017). La metodología de enseñanza. En R. Porlán (Coord.). Enseñanza universitaria. Cómo mejorarla (pp. 37-53). Madrid: Morata.

Finkel, D. (2008). Dar clase con la boca cerrada. Valencia: Universitat de València.

García, F. F. y Porlán, R. (2017). Los Principios Didácticos y el Modelo Didáctico Personal. En R. Porlán (Coord.). Enseñanza universitaria. Cómo mejorarla (pp. 93-104). Madrid: Morata.

Hernández Arredondo, E., García García, J., y López Calvario, C. (2019). Niveles de lectura de estudiantes de licenciatura: el caso de una tabla y una gráfica de líneas. Revista Digital: Matemática, Educación E Internet, 19(2). https: / /dol.org/10.18845/rdmei.v19i2.4214

Rivero, A. y Porlán, R. (2017). La evaluación en la enseñanza universitaria. En R. Porlán (Coord.). Enseñanza universitaria. Cómo mejorarla (pp. 73-91). Madrid: Morata

Jornadas de Formación e Innovación Docente del Profesorado | № 2 (2019) Esta obra se distribuye con la licencia Creative Commons Reconocimiento-NoComercial-SinObraDerivada 4.0 Internacional (CC BY-NC-ND 4.0.) 